\title{
La procédure de contrôle de l'attribution des marchés publics au Togo
}

\author{
Edem Kossivi Agbodji*
}

Le Togo a transposé dans son ordonnancement juridique, les dispositions de la directive $\mathrm{N}$ ${ }^{\circ} 04 / 2005 \mathrm{CM} / \mathrm{UEMOA}$ du 09 décembre 2005 portant procédure de passation, d'exécution et de règlement des marchés publics et des délégations de service public dans 1'Union Economique et Monétaire Ouest Africaine (UEMOA) et la directive $\mathrm{N}^{\circ} 05 / 2005 \mathrm{CM} / \mathrm{UEMOA}$ du 09 décembre 2005 portant contrôle et régulation des marchés publics et délégations de service public dans l'UEMOA. La mise en œuvre de ces dispositions a conduit, au plan institutionnel, à la création par la loi $\mathrm{N}^{\circ} 2009-13$ du 30 juin 2009 relative aux marchés publics et délégation de service public, des organes de passation, de contrôle et de régulation des marchés publics et délégation de service public. La réforme ainsi entamée s'est poursuivie par l'adoption du décret N²009-277/PR du 11 novembre 2009 portant code des marchés publics et délégation de service public. Les marchés publics et les organes de contrôle desdits marchés notamment ceux relatifs aux projets d'infrastructures au Togo sont donc soumis à un droit dont les principes sont, pour l'essentiel, édictés par les normes internationales et communautaires. Le contrôle des marchés publics est donc effectué à partir des principes fondamentaux de la commande publique et des réglementations issues des engagements internationaux. Ces principes et procédures désormais consacrés dans le nouvel ordonnancement juridique gouvernent la passation, l'attribution, l'exécution et le contrôle des marchés publics.

Les dispositions de l'article $1^{\mathrm{er}} \mathrm{du}$ décret $\mathrm{N}^{\circ} 2009-277 / \mathrm{PR}$ portant code des marchés publics et délégations de service public, définissent le marché public comme un " contrat écrit, conclu à titre onéreux, passé conformément aux dispositions de la loi $\mathrm{N}^{\circ} 2009-13 \mathrm{du}$ 30 juin 2009 relative aux marchés publics et délégations de service public, par lequel un entrepreneur, un fournisseur ou un prestataire de service s'engage envers l'une des personnes morales de droit public ou de droit privé visées dans ladite loi, soit à réaliser des travaux, soit à fournir des biens ou des services moyennant un prix ». On déduit de cette définition, que le contrôle des marchés publics consiste pour l'essentiel, en la vérification de l'application des principes gouvernant chaque étape de la procédure de passation desdits marchés. Le cadre normatif prévoit pour ainsi dire, une mise en œuvre obligatoire de règles aux étapes de préparation, de passation et d'exécution des marchés. Comment les marchés publics sont-ils attribués au Togo? Comment s'effectue le contrôle de l'attribution desdits marchés? Quels sont les organes en charge du contrôle desdits marchés?

Le Togo a opéré courant année 2009 une profonde réforme de son système des marchés publics, laquelle réforme avait pour objectif d'hisser le système togolais au rang des

* Magistrat. 
meilleures pratiques internationalement admises. Le droit des marchés publics au Togo est donc véritablement empreint des normes communautaires notamment les directives de l'Union Economique et Monétaire Ouest Africaine (UEMOA) régissant les marchés publics et des indicateurs de performance de l'Organisation de Coopération et de Développement Economique (OCDE).Comme tel, le droit positif des marchés publics au Togo présente,à l'instar des législations actuellement en vigueur dans les pays de la sous région, un caractère fortement international comme ont pu l'exprimer, à la phase de collecte des données ${ }^{1}$, les personnes en charge des marchés publics. Sur le plan pratique, eu égard au caractère encore nouveau de la matière, l'un des défis actuels est de faire maitriser les procédures de passation, d'exécution et de contrôle ainsi que la gestion des contentieux tant aux personnes responsables des marchés publics (PRMP) auprès de chaque autorité contractante, qu'aux candidats et soumissionnaires. C'est le lieu de relever que le droit positif des marchés publics au Togo est encore à ses débuts puisque son effectivité remonte à environ dix ans. Il s'agira donc ici de tenter d'appréhender, à l'épreuve de la pratique, la mise en œuvre, par les différents organes de contrôle, des règles prévues pour assurer plus de transparence et d'égalité des soumissionnaires dans l'attribution des marchés publics.

La présente analyse étudie sommairement le contrôle des marchés publics au Togo à la phase de leur attribution afin de mettre en exergue les règles gouvernant cette étape de préparation et de passation du marché pour mieux cerner le rôle des différents organes intervenant dans la procédure. Le choix d'encadrer l'analyse spécifiquement au contrôle exercé à la phase d'attribution du marché s'explique d'une part par le fait que le contrôle exercé à la phase d'exécution du marché s'effectue en cours ou quasiment à la fin de l'exécution du contrat ou du marché ${ }^{2}$; d'autre part ledit contrôle se limite et se confond dans une certaine mesure au contrôle de l'exécution de la Loi de finance.

Le contrôle des marchés publics à la phase d'attribution voit aussi intervenir la Cour des comptes, institution prévue par la constitution togolaise de 1992, et qui a été mise en place récemment pour satisfaire aux exigences de l'UEMOA. Il faudra alors certainement un peu plus de temps pour mieux apprécier l'efficacité du contrôle exercé par cette institution sur la transparence de l'attribution des marchés publics et sur l'économie du pays. Il aurait également pu être question dans la présente étude, du contrôle exercé par la Haute Autorité de Prévention et de Lutte contre la Corruption et les Infractions Assimilées (HAPLUCIA), créée par la loi $\mathrm{N}^{\circ} 2015$ du 28 juillet 2015, lequel organe planche actuellement sur la gestion des fonds alloués à la fédération togolaise de football dans le cadre de la Coupe d'Afrique des nations de 2013 et 2017 ainsi que sur le marché relatif au projet de bitumage de la route Lomé-Vogan-Anfoin. Seulement, le rapport des activités de cet organe

1 C'est le lieu de relever qu'avant la réglementation actuellement en vigueur, toutes les procédures relatives à l'attribution des marchés publics étaient gérées par une commission des marchés publics rattachée à la primature (cabinet du Premier ministre).

2 Ledit contrôle qui s'effectue a posteriori est quasi inopérant à cause du fait majoritaire et le critère de la mauvaise exécution n'est pas vraiment pris en compte lors de l'attribution de nouveaux marchés. 
n'est pas encore disponible afin qu'on puisse déduire des décisions prises, le type de contrôle exercé et les principes appliqués.

Faute d'avoir recueilli plus d'éléments et d'informations pratiques, le présent article n'abordera pas non plus le contrôle exercé par certaines structures ou institutions en l'occurrence l'inspection générale d'Etat rattachée directement à la présidence de la République et qui exerce un contrôle sur l'utilisation des deniers publics notamment le financement des projets d'infrastructures, l'inspection générale des finances et l'inspection du Trésor toutes deux placées sous l'autorité du ministre de l'économie et des finances ${ }^{3}$.

En évoquant finalement le principe de la séparation et de l'indépendance des fonctions de contrôle et de régulation des marchés publics ${ }^{4}$, l'analyse du contrôle de l'attribution des marchés publics au Togo peut s'effectuer d'une part,à travers les deux principaux points de contrôle que sont la Commission de Contrôle des Marchés Publics (CCMP) et la Direction Nationale de Contrôle des Marchés Publics (DNCMP); d'autre part, ledit contrôle fait intervenir les institutions de régulation et de gestion du contentieux que sont l'Autorité de Régulation des Marchés Publics (ARMP) et le juge administratif lesquelles institutions sont chargées de régler les différends et les recours dont ils sont saisis. Ce dispositif de contrôle des marchés publics au Togo s'inspire des directives de l'UEMOA et est arrimé sur les normes communautaires (A), privilégiant conséquemment la gestion et la régulation dans le règlement des contentieux $(\mathrm{B})$.

\section{A - Un contrôle encadré par les directives communautaires}

S'inspirant des directives de l'UEMOA, le droit positif des marchés publics au Togo consacre deux principaux points de contrôle de la passation et /ou de l'attribution des marchés publics. Il s'agit de la commission de contrôle des marchés publics (I) et de la direction nationale de contrôle des marchés publics (II).

\section{I- La Commission de Contrôle de Marchés Publics (CCMP)}

Aux termes des dispositions de l'article 9 du décret N²009-297/PR du 30 décembre 2009, une commission de contrôle des marchés publics (CCMP) est créée auprès de chaque autorité contractante et placée sous la responsabilité de la personne responsable des marchés publics (PRMP). Elle est chargée du contrôle a priori de la régularité de la procédure de passation des marchés publics et délégations de service public, depuis la phase de la planification jusqu'à l'attribution du marché ou de la délégation, et ce pour les marchés publics d'un

3 Ces structures contrôlent beaucoup plus l'utilisation des deniers publics et conséquemment l'exécution des marchés publics plutôt que leurs conditions d'attribution.

4 Le principe de la séparation et de l'indépendance des fonctions de contrôle et de régulation des marchés publics est issu des bonnes pratiques internationalement reconnues et destiné à améliorer la transparence, à renforcer l'efficacité de la lutte contre la corruption et à garantir des voies de recours efficaces. 
montant supérieur à un seuil fixé par voie règlementaire. Cette commission est composée de cinq membres désignés par l'autorité contractante.

Le contrôle exercé par la CCMP est un contrôle interne du processus de passation des marchés publics et le seuil financier qui détermine et enclenche tant sa compétence que son contrôle était compris entre 3 et 15 millions de francs CFA. Pour le compte l'année 2019 une nouvelle règlementation est entrée en vigueur et le contrôle interne de la CCMP s'effectue à partir d'un seuil financier compris entre 15 et 25 millions de francs CFA. Il s'agit donc de marchés d'importance moyenne. Dans sa mission de contrôle, la CCMP:

- procède à la validation du plan de passation de marchés de l'autorité contractante et des dossiers d'appel d'offres avant le lancement de l'appel à la concurrence et la publication correspondante;

- émet des avis de non objection et accorde les autorisations et dérogations nécessaires lorsqu'elles sont prévues par la réglementation en vigueur; à ce titre, elle exerce les mêmes compétences que la direction nationale de contrôle des marchés publics (DNCMP);

- procède à la validation du rapport d'analyse comparative des offres ou propositions et du procès verbal d'attribution provisoire du marché ou de la délégation approuvés par la commission de passation des marchés;

- procède à un examen juridique et technique du dossier du marché avant de le valider et au besoin, propose toute modification de nature à garantir la conformité du marché avec le dossier d'appel d'offres et la réglementation en vigueur;

- procède à la validation des projets d'avenants;

- établit à l'attention du représentant de l'autorité contractante un rapport annuel d'activités.

De manière générale la CCMP vérifie pour chaque marché, si la procédure de passation a été respectée. A cet effet, la PRMP envoie le dossier de l'offre à la Commission de Passation des Marchés Publics (CPMP) pour ouverture et analyse; la CPMP effectue une évaluation technique (notamment la vérification du respect des caractéristiques) et financière (la détermination du moins ou du mieux disant) et dresse un procès verbal d'évaluation qu'elle transmet à la PRMP, laquelle à son tour la communique à la CCMP. Cette dernière suite à son contrôle, émet soit un avis de non objection et le marché est attribué au soumissionnaire retenu, ou elle émet un avis d'objection entrainant une reprise de la procédure.

\section{II - La Direction Nationale de Contrôle des Marchés Publics (DNCMP)}

La DNCMP est chargée d'un contrôle a priori et procède également a posteriori au contrôle des procédures de passation des marchés d'un montant inférieur au seuil déterminant son contrôle a priori. Le seuil financier déterminant la compétence et le contrôle a priori de la DNCMP était de 25 millions FCFA jusqu'à fin 2018. Depuis l'année 2019, le seuil est désormais fixé à 85 millions FCFA. Le contrôle a priori de la DNCMP se fait donc sur les projets soumis à la procédure d'appel d'offre (pour les marchés d'un montant supérieur à 
85 millions). On peut déduire de cette nouvelle réglementation, la volonté du gouvernement togolais de donner plus de champ et de latitude à chaque autorité contractante qui dispose à ses côtés comme nous l'avons précédemment présenté, d'une CCMP; c'est dire que le contrôle interne de la CCMP se retrouve élargi entrainant plus de célérité et de sérénité dans les procédures de passation.

Avec l'actuelle réglementation, les projets d'infrastructures soumis à une procédure de demande de cotation (pour les marchés d'un montant inférieur ou égal à 10 millions), ou de demande de renseignement de prix (pour les marchés d'un montant supérieur à 10 millions mais inférieur à 85millions) relèvent de la compétence de la CCMP s'agissant de leur contrôle a priori. Le contrôle de la DNCMP se fait à l'externe et a posteriori sur lesdits marchés.

La DNCMP surveille également l'activité des directions régionales chargées du contrôle des marchés publics. Elle assure les missions de suivi de l'exécution des marchés publics et des délégations de service public ${ }^{5}$. S'agissant spécifiquement du contrôle a priori, la procédure de contrôle est quasiment similaire à celle de la CCMP. En effet, la PRMP élabore le dossier d'appel d'offre et l'envoie à la DNCMP pour avis; si la DNCMP émet un avis défavorable, le dossier retourne à la PRMP pour correction du dossier d'appel d'offre (par exemple, la PRMP a introduit dans le dossier d'appel d'offre des critères discriminatoires que les vérifications des caractéristiques ont révélés). Dans le cas où la DNCMP émet un avis favorable, l'appel d'offre est lancé et publié pour une période d'un mois dans le quotidien national (Togo presse). A l'issue de la période ouverte pour les offres, la CPMP procède à l'ouverture et à l'évaluation des offres; une commission d'analyse des offres est mise en place en lieu et place de la commission de passation des marchés publics (CPMP) lorsque le domaine ou le projet nécessite des connaissances techniques qui échappent aux membres de la CPMP. Après l'ouverture et l'analyse des différentes offres, le dossier et le procès verbal d'évaluation sont envoyés à la PRMP qui les transmet à son tour à la DNCMP pour contrôle et avis. La DNCMP reprend le dossier, l'analyse et l'évalue comme l'avait étudié la commission d'analyse et émet finalement son avis de non objection. Après ledit avis, la PRMP écrit à tous les soumissionnaires et les informe de l'attributaire du marché et du prix auquel le marché a été attribué.

Les autres soumissionnaires disposent d'un délai de 15 jours pour les éventuels recours adressés à la PRMP ou à l'ARMP. Il convient de préciser que le recours adressé à la PRMP à la suite de l'attribution d'un marché, n'est pas un recours gracieux; au contraire, ce recours marque le début de la phase contentieuse et entraine l'arrêt de la procédure d'attribution et d'exécution du marché. La PRMP informe, le cas échéant, tous les autres soumissionnaires du recours formulé et de l'arrêt de la procédure jusqu'au règlement définitif. Si suite au recours devant la PRMP qui dispose d'un délai de réponse, le requérant obtient gain de cause, le marché est attribué à ce dernier; dans le cas contraire l'attributaire initial

5 Article 2 du décret $\mathrm{N}^{\circ} 2009-295 / \mathrm{PR}$ portant missions, attributions, organisation et fonctionnement de la DNCMP. 
est conservé. C'est dire que si la PRMP constate déjà à son niveau que le recours est fondé, le marché peut être attribué au requérant. Si le requérant n'est pas satisfait de la suite donnée par la PRMP à sa requête pour l'avoir rejetée comme mal fondée et conservé l'attributaire initial, le requérant peut saisir l'ARMP.

\section{B - Un contentieux partagé entre régulation, gestion et règlement}

$\mathrm{Au}$ Togo, la transposition des directives de l'UEMOA dans la réglementation interne s'est traduite par la mise en place d'une institution à compétence centrale, l'Autorité de Régulation des Marchés Publics (ARMP), (I), laquelle partage avec le juge administratif, la gestion des contentieux (II).

\section{I- L'Autorité de Régulation des Marchés Publics (ARMP) : entre régulation et règlement non juridictionnel}

L'objectif visé à travers la mise en place de l'Autorité de Régulation des Marchés Publics (ARMP) est de doter le système des marchés publics d'un dispositif institutionnel performant, d'encourager la professionnalisation des acteurs de la commande publique, de renforcer la lutte contre la corruption et de garantir des voies de recours efficaces et rapides. De façon générale, il s'agit de conférer une plus grande souplesse et adaptation aux impératifs économiques de la mise en œuvre des fonctions de régulation initialement dévolues à l'administration centrale. Le rôle de l'ARMP, convient-il de le préciser, est d'instaurer un environnement de concurrence et de transparence en éliminant toutes les restrictions anormales à la participation d'opérateurs privés dans les secteurs régulés et veiller à la détermination des règles et procédures d'attribution des marchés et des concessions. L'ARMP a donc pour missions:

- de veiller à la mise en application de la réglementation et de faire au gouvernement des propositions de nature à améliorer et renforcer l'efficacité du système;

- d'élaborer, de diffuser et de mettre à jour les documents types, manuels de procédures, guides d'évaluation;

- de constituer une banque de données sur le système;

- d'évaluer des capacités;

- de former, d'informer et de sensibiliser;

- de contrôler les procédures de certification des entreprises;

- la mise en œuvre d'audits annuels indépendants techniques et/ou financiers

- d'initier des enquêtes portant sur la transparence des procédures de passation et d'exécution;

- de prononcer les sanctions des irrégularités constatées;

- le règlement non juridictionnel des litiges nés de la passation des marchés publics et des délégations de service public. 
L'ARMP est composé de trois organes: le conseil de régulation, le comité de règlement des différends et la direction générale.

Le comité de règlement des différends (CRD) a pour mission de statuer, au terme d'une procédure équitable et contradictoire, sur les litiges opposant soit une autorité contractante et la DNCMP, soit des candidats et des soumissionnaires, soit une autorité contractante ou la DNCMP et un candidat ou un soumissionnaire. Le CRD est composé de façon tripartite et paritaire de trois membres du Conseil de régulation provenant de l'Administration, du secteur privé et de la société civile, désignés par le Conseil pour une période de trois ans non renouvelables. Les décisions rendues par le CRD peuvent faire l'objet d'un recours judiciaire dans un délai de cinq jours ouvrables à compter de la date de notification de la décision faisant grief.

Conformément aux directives de l'UEMOA, la loi relative aux marchés publics et délégations de service public et le code des marchés publics instituent une procédure de recours suspensif au stade de passation de marchés. Ce recours doit au préalable être exercé auprès de l'autorité contractante comme exposé précédemment. Une copie du recours adressé à l'autorité contractante est communiquée à l'ARMP; ce recours peut porter sur:

- la décision prise en matière de pré qualification ou d'établissement de la liste restreinte;

- la décision d'attribuer ou de ne pas attribuer le marché ou la délégation;

- les conditions de publication des avis;

- les règles relatives à la participation des candidats et aux capacités et garanties exigées;

- le mode de passation et la procédure de sélection retenue;

- les spécifications techniques retenues;

- les critères d'évaluation.

Pour être recevable, le recours devant le CRD doit s'exercer dans les conditions suivantes:

- le requérant doit avoir intérêt à agir;

- le recours préalable doit avoir été fait;

- le recours doit être formé dans le délai légal.

Sur le fondement des informations recueillies dans l'exercice de ses missions ou de toute information communiquée par des autorités contractantes, des candidats soumissionnaires ou des tiers, l'ARMP peut aussi se saisir d'office à la demande de son président ou du tiers $(1 / 3)$ de ses membres et statuer sur les irrégularités, fautes et infractions constatées. Cette saisine d'office de l'ARMP est suspensive de la procédure d'attribution définitive du marché ou de la délégation.

De manière pratique, lorsque l'ARMP est saisie, elle informe la PRMP du recours et sollicite communication de l'ensemble du dossier ${ }^{6}$. Une fois que la PRMP envoie le dossier à l'ARMP, le CRD siège et étudie le dossier. Si le CRD donne gain de cause au requérant, il

6 Le dossier devra comporter: le rapport d'évaluation, le dossier d'appel d'offre, les offres des soumissionnaires et un mémoire. Ce recours (saisine d'office de l'ARMP) peut être exercé quelque soit le mode de passation choisi notamment la consultation restreinte. 
écrit à la PRMP l'informant de sa décision et de ce que le marché est attribué au requérant. Si le recours du requérant est déclaré non fondé, le comité informe également la PRMP et la procédure reprend avec la confirmation de l'attributaire initial. Si le soumissionnaire ayant saisi l'ARMP estime que la décision du CRD ne lui convient pas, il saisit le juge administratif.

Le CRD rend ses décisions dans un délai de sept jours ouvrables et sa décision est immédiatement exécutoire. Elle peut faire l'objet d'un recours devant la chambre administrative de la Cour suprême. Ce recours n'est cependant pas suspensif.

\section{II - Le juge administratif}

Aux termes des dispositions de l'article 127 du code des marchés publics, les marchés publics étant des contrats administratifs, la compétence de principe est celle du juge administratif en cas de recours contre une décision du CRD. Le juge administratif contrôle ainsi l'application effective des règles et directives gouvernant l'attribution des marchés. On peut structurer en trois parties le contentieux des marchés publics devant le juge administratif:

- le recours pour excès de pouvoir

- l'action en annulation du marché

- l'action en responsabilité administrative

S'agissant d'abord du recours pour excès de pouvoir, c'est la chambre administrative de la Cour suprême qui est compétente en premier et dernier ressort. Il en est ainsi par exemple des actes détachables du marché public ou de la délégation de service public comme: l'autorisation de contracter, la décision de contracter ou de ne pas contracter, l'opération d'attribution, l'approbation du contrat. Ce recours peut être exercé par un soumissionnaire qui présentera devant le juge administratif des moyens de fait et de droit susceptibles d'établir l'illégalité de l'acte attaqué. Si l'acte détachable est le support indispensable du contrat passé, le marché attribué en vertu dudit acte détachable est lui-même potentiellement entaché d'illégalité. L'annulation de l'acte détachable peut avoir pour conséquence la nullité du contrat passé lorsque cette nullité résulte d'une illégalité grave comme par exemple le défaut d'approbation ou de qualité de l'autorité signataire qui n'avait plus compétence pour signer le contrat.

S'agissant ensuite de l'action en annulation du marché, l'article 29 de la loi N²009013 du 30 juin 2009 relative aux marchés publics et délégations de service public précise que tout soumissionnaire évincé peut demander dans les trois mois de la publication de tout contrat ou avenant, leur annulation devant la juridiction administrative compétente. Le soumissionnaire évincé devra nécessairement établir une irrégularité se rapportant à des faits de corruption ou de fraude ou encore de manière plus générale, à une violation grave des dispositions et principes de la réglementation applicable en matière de marchés publics et délégations de service public.

S'agissant enfin de l'action en responsabilité administrative, l'article 30 de la loi $\mathrm{N}$ ²009-013 du 30 juin 2009 relative aux marchés publics et délégations de service public 
précise que toute personne qui a subi un dommage résultant d'un acte de corruption ou d'une violation des dispositions de la réglementation applicable en matière de marchés publics ou délégations de service public peut intenter une action en indemnisation contre l'Etat et toute autre personne physique ou morale impliquée, en vue d'obtenir la réparation de l'intégralité de ce préjudice; cette réparation pouvant porter sur les dommages patrimoniaux subis, le manque à gagner et les préjudices extrapatrimoniaux. Le requérant devra nécessairement établir:

- la faute résultant des agissements de l'autorité contractante;

- le préjudice subi;

- le lien de causalité entre la faute et le préjudice.

Pour des questions liées à la solvabilité, il est plus opportun pour le requérant de rechercher la responsabilité administrative de l'autorité contractante de rattachement. Cette dernière, en cas de condamnation, pourra se retourner contre l'agent fautif au moyen d'une action récursoire. 